\title{
Enhancing miRNAs Capture on Polydimethylsiloxane Surface with Nanostructuration
}

Patrizia Guida ${ }^{1 \# *}$, Roberto Lo Savio ${ }^{1 \#}$, Cristina Potrich ${ }^{2,3 \#}$, Valentina Vaghi ${ }^{2}$, Elena Angeli ${ }^{1}$, Lia Vanzetti ${ }^{4}$, Denise Pezzuoli ${ }^{1}$, Laura Pasquardini ${ }^{2,5}$, Giuseppe Firpo', Luca Repetto ${ }^{1}$, Diego Repetto', Cecilia Pederzolli ${ }^{1}$ and Ugo Valbusa ${ }^{1}$

${ }^{1}$ Nanomed Laboratories, Dipartimento di Fisica, Università di Genova, Genova, Italy

${ }^{2}$ Fondazione Bruno Kessler, Center for Materials and Microsystems, LaBSSAH, Povo (Trento), Italy

${ }^{3}$ CNR- Institute of Biophysics, Unit at Trento, Povo (Trento), Italy

${ }^{4}$ Fondazione Bruno Kessler, Center for Materials and Microsystems, MNF-MateC, Povo (Trento), Italy

${ }^{5}$ Department of Industrial Engineering, University of Trento, Povo (Trento), Italy

\#Contributed equally

\begin{abstract}
MicroRNAs (miRNAs) modulate gene expression at post-transcriptional level, while their aberrant presence in circulation correlates with the most common human disorders such as cancer, neuro-degenerative and immune-related diseases. Currently, the pre-concentration of such important bio-markers present at low concentrations in biological fluids, which would make their identification and quantification easier, remains a challenging issue for biosensor-based non-invasive analyses. This paper describes a new nanostructure-based polymeric platform for enhancing adsorption capability of microRNAs, such as the cancer-associated miRNA-21. In this purification strategy, a nano-hole pattern was manufactured by Replica Molding (REM) and fabricated on Polydimethylsiloxane (PDMS) large-areas. Interestingly, the microRNAs adsorption is resulted favoured by this proper topography. In planning to concentrate the patient's miRNAs used as biomarkers, PDMS surfaces with nanostructures were not further coated with any adhesive molecules to prevent forced surface-biomolecule adhesive regions and evaluate the mere influence of the surface topography and the ionic microenvironment. Both the nanopattern and the solution ionic strength promote adsorption of miRNAs by a sensitive and efficient method, which do not need probe immobilization, enzymatic reaction or further treatments. These studies revealed a two-fold higher fluorescent signal after solid-phase purification protocol compared to standard PDMS obtained by spin-coating. While this paper focuses on nanostructure-miRNA adsorption, the general strategy of trapping miRNAs on nano-hole patterns should be broadly applicable for purification of other miRNAs through microfluidic biosensors and should have basic as well as clinical research applications.
\end{abstract}

Keywords: Polydimethylsiloxane; Large-area Nanostructuring; Labon-a-chip; Oncology; Body fluids; Biomarkers Purification; miRNA

\section{Introduction}

In the last years, predictive and preventive medicine took advantage on the novel discoveries in nano-diagnostics and smart therapies, mainly based on the specific identification of molecular biomarkers, such as nucleic acids (DNA, miRNA) and proteins. The possibility to detect low amount of biomarkers directly in biological fluids gives a great advantage in the early diagnosis and therapy of malignant diseases, which have high social impact. In fact, a correlation between degenerative, contagious, or inflammatory illnesses with the presence of specific biomarkers like miRNAs is now well established [1-5]. However, this remains a challenging task since in each biological sample several different biomolecular species co-exist, and moreover it is very hard to treat them in order to achieve the degree of purity and concentration required for a quantitative, specific, and reliable identification. To overcome these obstacles, several nanotechnologybased systems are being developed and tested for miRNAs detection. Nanobiosensor development is particularly relevant for such miRNAs, whose aberrant expression has been shown to correlate with the pathogenesis and progression of several diseases including cancer [6,7]. MicroRNA-21 (miR-21), in particular, is largely studied since is overexpressed in a variety of solid $[8,9]$ and hematological tumors $[10,11]$. For these reasons, miR-21 was selected as a microRNA prototype in this study.

Recently, it has been proposed that nanotechnology-based approaches [12-14] alternative to conventional methods [15-19] for miRNA detection and quantification can be developed using innovative biosensors made of Polydimetylsiloxane (PDMS) [20-22]. PDMS is known to have numerous advantages, supporting its development as a useful platform for biosensor and biomedical applications [20,23-25]. Particularly, the attractiveness of such polymer lies in the fact that its chemical and physical properties and also the surface topography can be modulated [26]. These features combined with the manufacturing of biomolecule-specific topographies contribute to the improvement of PDMS performance in terms of sensitivity and efficiency. Recently, the fabrication of PDMS-based devices has introduced significant advances in several analytical techniques in terms of sensitivity, time consuming, and sample volume. Since PDMS material revealed characteristic properties and reliability, PDMS-nanotechnology has therefore gained great attention in developing new generation of research tools. However, both traditional and technology-based approaches have yet limitations mainly related to sample processing, purification, separation and enrichment of the small RNA fraction [12,27]. In fact, advanced biosensors still require specific conjugation steps [28] and expensive equipment which need to be overcome before they can have a real clinical application.

PDMS nanostructures manufacturing may be useful to enhance the efficient purification of miRNA circulating bio-markers present at low

*Corresponding author: Patrizia Guida, Physics Department, Nanomed Laboratories, University of Genova Via Dodecaneso, 33, 16146 Genova, Italy, Tel: 390103536267; E-mail: patrizia.guida@unige.it

Received: April 13, 2017; Accepted: April 24, 2017; Published: April 28, 2017

Citation: Guida P, Lo Savio R, Potrich C, Vaghi V, Angeli E, et al. (2017) Enhancing miRNAs Capture on Polydimethylsiloxane Surface with Nanostructuration. J Nanomed Nanotechnol 8: 437. doi: 10.4172/2157-7439.1000437

Copyright: $\odot 2017$ Guida $\mathrm{P}$, et al. This is an open-access article distributed under the terms of the Creative Commons Attribution License, which permits unrestricted use, distribution, and reproduction in any medium, provided the original author and source are credited. 
concentrations in most specimen such as tissue biopsies and body fluids $[29,30]$. This is particularly useful for polymeric platforms that perform purification processes, which can be improved with appropriate nanostructured surfaces. In particular, prior works on these PDMS platforms, have shown that the adsorption of miRNA molecules can be increased by random roughening present on the sensing surface [21]. With the aim of improving the miRNA adsorption, we developed a proper nanostructuration of the PDMS to increase the surface-tovolume ratio and, furthermore, to get a topography as conform as possible to miRNAs molecules. The main advantages of our fabrication protocol are: (i) engineering of material at the nanoscale is achieved through quick and not expensive methodologies; (ii) nanopatterns are obtained on large-area compared to standard technologies like focused ion beam machining or reactive ion etching.

In this paper, we conduct specific experiments testing the adsorption efficiencies for miRNA molecules captured without any specific immobilized probes on conformingly nanostructured surfaces. Functional assay analysis revealed that the miRNA adsorption was increased by one order of magnitude.

The described results provide direct evidence that the size and topography of the nanostructures present on PDMS surfaces influence the miRNA adsorption and facilitates solid-phase extracellular miRNAs purification from samples.

\section{Materials and Methods}

\section{PDMS pattern fabrication}

The Polydimethylsiloxane (PDMS, SYLGARD ${ }^{\circ} 184$ Silicone Elastomer, Dow Corning) surface with nano-holes patterns was fabricated starting from the nanoparticles that intrinsically assemble from borosilicate glass productions (Supplementary Information). The surface of coverslip of $24 \times 40 \mathrm{~mm}$ (Corning Borosilicate Cover glass Cat. No. 2975-244, Thickness 1), having such gripped nanoparticles (Supplementary Figure S1), was used to generate large-area PDMS replicas of $10 \mathrm{~cm} 2$ by Replica Molding. We tested two different PDMS Replica Molding conditions (named sample I and sample II) both allowing to obtain desired holes pattern. Before using, coverslips were firstly cleaned in a dry nitrogen stream, sterilised with $70 \%$ (v/v) ethanol for 10 minutes, repeated three times, then dried in a nitrogen stream followed by further 10 minutes in the oven at $60^{\circ} \mathrm{C}$. Coverslips were functionalized as previously described [26]. Briefly, coverslips were firstly treated with oxygen plasma (Tucano plasma reactor Gambetti Kenologia) at $50 \mathrm{~W}$ RF power, for $60 \mathrm{sec}$ with an $\mathrm{O} 2$ flux of $15 \mathrm{sccm}$ and 2.0E-01 mbar; then, coverslips were exposed to a 30 minutes long vapour phase deposition of $20 \mu \mathrm{l}$ (sample I) and of $40 \mu \mathrm{l}$ (sample II) of Trichloro $(1 \mathrm{H}, 1 \mathrm{H}, 2 \mathrm{H}, 2 \mathrm{H}$-perfluorooctyl) silane (FOTS, 448931-10G, Sigma-Aldrich, Saint Louis, Missouri, USA) in vacuum. PDMS was used with a formulation of 10:1 w/w of the elastomer to curing agent. The mixture was vacuum-degassed for 20 minutes prior dipping onto the functionalized glasses. The PDMS curing step was started at room temperature for $1 \mathrm{~h}$ and was completed in an oven for $2 \mathrm{~h}$ at $65^{\circ} \mathrm{C}$. Finally, the cured PDMS is peeled off and used as a patterned surface.

\section{Characterization of capturing surfaces}

Topography of PDMS replicas was investigated by atomic force microscopy (AFM) (Dimension 3100 Veeco, hybrid XYZ head, Santa Barbara, CA, USA; and Olympus OMCL-AC160 tips nominal apical radius $<10 \mathrm{~nm}$, Olympus corporate, Tokyo), operating in tapping mode under ambient conditions. The root mean square (RMS) value of the roughness height in native PDMS was of $0.47 \pm 0.06 \mathrm{~nm}$, in accordance with literature measurements [31,32]. Likewise, the PDMS replica was characterized by X-ray photoelectron spectroscopy (XPS) to evaluate the chemical composition of the layers which directly interact with the biomarkers. XPS measurements were performed using a Scienta ESCA200 instrument equipped with a hemispherical analyzer and a monochromatic $\mathrm{Al} \mathrm{Ka}(1486.6 \mathrm{eV}) \mathrm{X}$-ray source, in transmission mode. The emission angle between the analyzer axis and the sample surface was $30^{\circ}$, corresponding to a sampling depth of approximately $5 \mathrm{~nm}$ [33]. For each sample Si 2 p, O 1s, C 1s, and $\mathrm{F}$ 1s core lines were recorded, using the low electron energy flood gun to compensate sample charging. The quantification, reported as relative elemental percentage, was done by using the integrated area of the fitted core lines, after Shirley background subtraction, and by correcting for the atomic sensitivity factors. This procedure provides a quantitative analysis.

\section{Functional assay of capturing surfaces}

10 ng of synthetic microRNA (hsa-miR-21-Alexa488; 5'-UAGCUUAUCAGACUGAUGUUGA-3') were incubated on the PDMS surfaces for $20 \mathrm{~min}$ at room temperature, washed with ultrapure water and imaged at the fluorescence microscope Leica DMLA (LeicaMicrosystems, Germany), equipped with a mercury lamp and fluorescence filter L5 (Leica Microsystems, Germany). All surfaces were observed with a $20 \times$ magnification objective and measured with a cooled CCD camera (DFC 420C, Leica Microsystems, Germany). All fluorescence images were analyzed with Fiji software [33] in order to quantify the fluorescence of the adsorbed RNA.

\section{Results and Discussion}

The use of microdevices as lab-on-a-chip (LOC) for clinical applications implies to process a small amount of biological sample where the molecules target of the analysis is dispersed in little quantity. Moreover, a LOC have to be easy-to-used and compact, a requisite needed to process the sample also in non-specialized environments, such as decentralized medical structures or by non-trained personnel. To fulfil these requirements, a new strategy is presented in this paper. PDMS nanostructured surfaces were prepared via replica moulding starting from selected salinized coverslips. The new surfaces, possibly implementable in future LOCs, were deeply characterized, tested for microRNAs capture and their performances compared to nonnanostructured surfaces, as discussed below.

\section{Strategy of miRNA-conformed surface fabrication}

Physical dimensions of miRNAs are extremely small. The estimated length of persistence of a single-chain nucleic acid having similar number of nucleotides of miRNA-21 (which has $22 \mathrm{ntd}$ ), was of 0.4$0.7 \mathrm{~nm}[34,35]$. Based on miRNA molecules dimensions, we attempted to fabricate a nano-hole pattern of about $5 \mathrm{~nm}$ in depth to obtain both improved surface to volume ratio and a size range similar to the tested molecules. The strategy assumed in this study is summarized in Figure 1, and the PDMS surfaces with nano-holes patterns, obtained as described above, are show in Figure 2.

The interaction between biomolecules and materials is influenced strongly by their chemical composition and morphology at the surfaces of the materials [36]. In order to investigate the factors that affect miRNA adsorption on PDMS surfaces, we used a combination of XPS and AFM analyses. In particular, the hypothesis focuses on the suitability of the nano-hole sizes and on the chemical composition of the interacting surface that induces a favourable microenvironment to miRNA binding. XPS measurements showed the presence of fluorine 
Citation: Guida P, Lo Savio R, Potrich C, Vaghi V, Angeli E, et al. (2017) Enhancing miRNAs Capture on Polydimethylsiloxane Surface with Nanostructuration. J Nanomed Nanotechnol 8: 437. doi: 10.4172/2157-7439.1000437

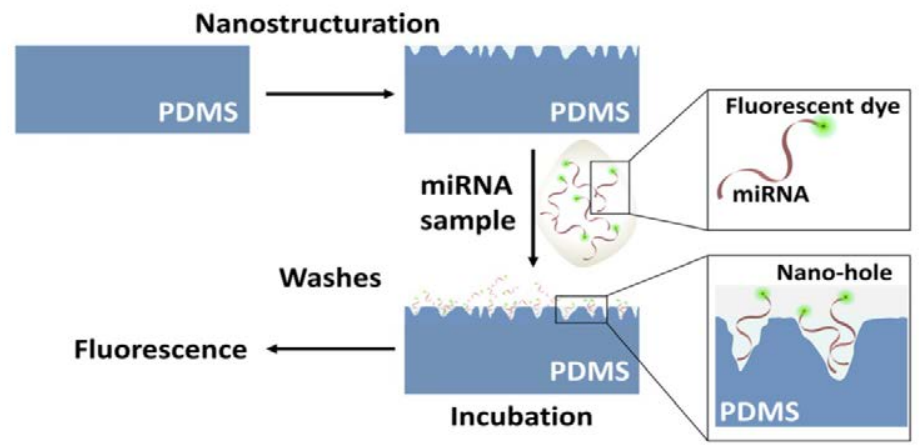

Figure 1: Schematic illustration of the nano-patterned surface used as capturing layer for solid-phase miRNA adsorption. A fluorescently-labelled microRNA (miR-21 conjugated with Alexa488) was incubated on the surface for $20 \mathrm{~min}$. After several washes with ultrapure water aimed at removing the unbound miRNA, the surfaces were imaged at the fluorescence microscope.

A

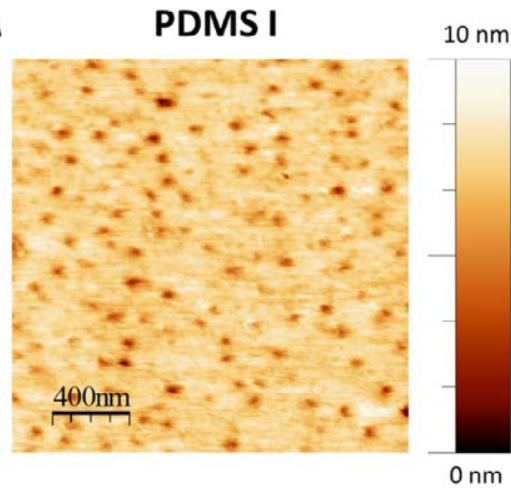

C

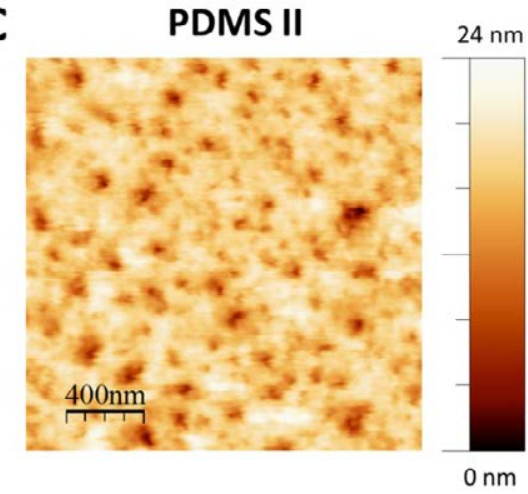

B

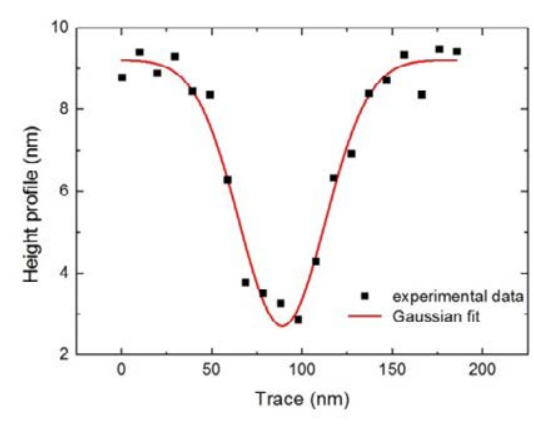

D

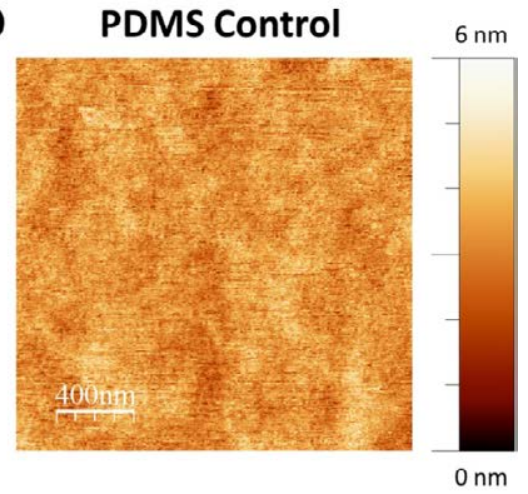

Figure 2: AFM topography of the surfaces of PDMS nano-hole pattern (A) obtained by first functionalization procedure (I); (B) profile of a nano-hole from sample I; (C) topography of pattern obtained by produre II; (D) Control is a native PDMS surface. The higher contamination of FOTS prevented the (C) pattern to be used. All scans are $2 \times 2 \mu \mathrm{m}$.

on PDMS replicas made on the functionalized glasses (Table 1), confirming that some silane molecules can be transferred to the PDMS replicas. In particular, the sample II presented the highest fluorine percentage as expected for the use of the double silane quantity in the functionalization protocol. Beside fluorine, the chemical composition has a relative percentage of carbon, oxygen and silicon compatible with the standard composition of PDMS, as reported in the literature $[31,33,37]$ and evident for the control surface, which is native PDMS and did not received any functionalization step.

The AFM images reported in Figure 2 clearly show the formation of the nano-holes pattern; while the surface of the native PDMS is smooth and does not evidence any topographic feature. In the patterned samples the nanometric holes are well visible and the inset in Figure 2B aims to show a profile of a single nano-hole from sample I. In order to extract quantitative information about the holes distribution, we evaluated the average width and depth of the holes. The profile of 50 random holes was extracted using the Gwyddion software [38] and fitted with a Gaussian profile. The average width and depth of the holes in sample I are $40 \pm 10$ and $4.5 \pm 1.0 \mathrm{~nm}$, respectively; as expected, the holes in the sample fabricated using higher silane concentration are wider and deeper, with corresponding average values of $73 \pm 23$ and $10.6 \pm 2.8 \mathrm{~nm}$. 
Citation: Guida P, Lo Savio R, Potrich C, Vaghi V, Angeli E, et al. (2017) Enhancing miRNAs Capture on Polydimethylsiloxane Surface with Nanostructuration. J Nanomed Nanotechnol 8: 437. doi: 10.4172/2157-7439.1000437

Page 4 of 5

\begin{tabular}{|c|c|c|c|c|}
\hline Surface & O (\%) & C (\%) & Si (\%) & F (\%) \\
\hline Control & 23.0 & 42.8 & - & 34.2 \\
\hline Sample I & 24.9 & 38.9 & 26.1 & 3.1 \\
\hline Sample II & 21.4 & 34.1 & 17.9 \\
\hline
\end{tabular}

Table 1: Elemental composition (atomic percentage) determined by XPS analysis at $30^{\circ}$ on differently prepared PDMS replicas. The standard error does not exceed the $1-2 \%$ of the reported value. Control surface is a PDMS replica from non-functionalized glass.

As shown in Figure 3, the presence of proper nanopatterns (bars I and II) can positively affect the miR-21 capturing. This performance was possible when nanostructures and biomolecule have comparable dimensions, as in accordance with the nanostructures' properties of influencing biomolecules behaviour described in literature [36,39]. The presence of conforming nano-hole pattern is an improvement with respect to the random roughness present on a PDMS surface obtained by spin-coating (bar "S", Figure 3), a surface that we previously studied for RNA capture from biological samples [21,31]. The microRNA capture by the nanopatterned surfaces was indeed more than doubled with respect to the previous PDMS surface. This aspect is particularly relevant considering the low abundance of microRNAs used as biomarkers and dispersed in body fluids. A doubling in the surface capturing efficiency is a real improvement that goes in the direction of designing microdevices with nanopatterned surfaces, with a possible huge increase in the biomarker capture. As note, although native hydrophobic PDMS (untreated) is known to interact with polar samples [40], stronger interactions were observed when nanostructuration was present. Finally, Figure 3 also shows that the high presence of fluorine onto the capturing surface reduce the nanostructures effect (bar II). An example of fluorescently-labelled miR-21 adsorbed on the different surfaces is shown in Supplementary Figure S2.

Our scope was to increase platform sensitivity and biomarker capture efficiency by increasing the number of binding events and the surface area. Looking forward, the implemented performance described could allow to overcome the bottlenecks of the surface devices currently used both in experimental and clinical environments. So that, the fabrication of a conforming active region of the platform, i.e. the part responsible for the capture of biomarkers, substantially introduce new interesting prospective for PDMS-based platforms applied to biomarker detection. Our results are in accordance with established nanomaterials special properties which not only allow to reduce the systems' size, but also add extra useful characteristics to biosensor's surfaces leading to effective improvements in efficiency and sensibility of analysis result $[12,41]$. Remarkable surface to volume ratio and a size range similar to the biomolecules are responsible for efficient inter-reactions between nanomaterials and biomarkers [41]. Importantly, large active areas allow to increase the volume of biological liquid samples that can be analyzed by the biosensors, capturing very low-concentrated biomarkers. Although the described approach still needs to be optimized, the obtained topography resulted in an efficient and affordable system to improve biomarker capture from biological samples, laying the basis for the implementation of microdevices designed with a suitable surface topography.

\section{Conclusions}

As the demands of nanostructured polymers have been increased in biomedical applications due to their new properties and performance, attempting to fabricate proper nanopatterns has become increasingly more important. The PDMS roughness is dependent on the processing and therefore, directly dependant on the molding process as PDMS precisely copies even the nanometric features. Increasing the surface roughness means to increase the surface area and thus, larger area for

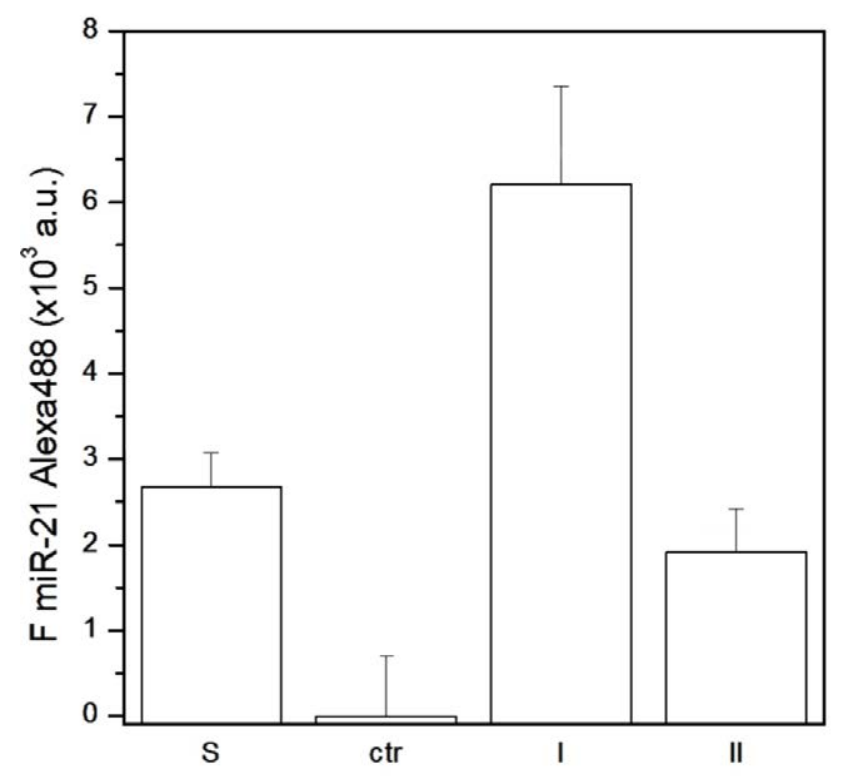

Figure 3: Functional assay on the PDMS surfaces. $10 \mathrm{ng}$ of miR-21 A488 were incubated on the different PDMS replicas: control surface (ctr; replica from non-functionalized glass) and two replicas from functionalized glass (replica II have the double of silane in the functionalization protocol). Surface named $S$ (spin PDMS) represents the starting surface to be ameliorated for its miRNAs capture.

interaction/adsorption. Moreover, in this work we provides evidence that proper surface topography plays an important role in miRNA-21 biomolecule-surface interaction, reporting the role of nano-hole pattern played in doubling the effects of previously random nanostructured surface on the capture of miRNA molecules. The nanopatterned surface with increased capturing efficiency was fabricated by affordable replica molding procedure on PDMS large surface.

Looking forward, the physicochemical properties of PDMS pattern could be further tuned, including the optimisation of surface chemistry, to enhance their selectivity performance. This nano-holebased miRNA purification technique could be further improved and validated for innovative, easy-to-use and integrated device for the efficient purification of less abundant circulating microRNAs for early diagnosis of diseases.

\section{Acknowledgement}

This work was supported by grants from the Italian Ministry of Education University and Research, Flagship Project Nanomax and FIRB project Newton RBAP11BYNP_003, U.V

\section{References}

1. Roberts APE, Lewis AP, Jopling CL (2011) The role of MicroRNAs in viral infection. Prog Mol Biol Transl Sci 102: 101-139.

2. Ha T-Y (2011) MicroRNAs in Human Diseases: From Lung, Liver and Kidney Diseases to Infectious Disease, Sickle Cell Disease and Endometrium Disease. Immune Netw 11: 309-23. 
Citation: Guida P, Lo Savio R, Potrich C, Vaghi V, Angeli E, et al. (2017) Enhancing miRNAs Capture on Polydimethylsiloxane Surface with Nanostructuration. J Nanomed Nanotechnol 8: 437. doi: 10.4172/2157-7439.1000437

3. Mi S, Zhang J, Zhang W, Huang RS (2013) Circulating microRNAs as biomarkers for inflammatory diseases. MicroRNA (Shāriqah, United Arab Emirates) 2: 63-71.

4. Ardekani AM, Naeini MM (2010) The role of microRNAs in human diseases. Avicenna J Med Biotechnol 2: 161-179.

5. Basavaraju M, Lencastre A de (2016) Alzheimer's disease: presence and role of microRNAs. Biomol Concepts 7: 241-252.

6. Calin GA, Croce CM (2006) MicroRNA signatures in human cancers. Nat Rev Cancer 6(November) 857-866.

7. Mulrane L, McGee SF, Gallagher WM, O'Connor DP (2013) miRNA dysregulation in breast cancer. Cancer Res 73: 6554-6562.

8. Taylor DD, Gercel-Taylor C (2008) MicroRNA signatures of tumor-derived exosomes as diagnostic biomarkers of ovarian cancer. Gynecol Oncol 110: 13-21.

9. Wu J, Li G, Wang Z, Yao Y, Chen R, et al. (2015) Circulating MicroRNA-21 is a potential diagnostic biomarker in gastric cancer. Dis Markers.

10. Li J, Fu R, Yang L, Tu W (2015) miR-21 expression predicts prognosis in diffuse large B-cell lymphoma. Int J Clin Exp Pathol 8: 15019-24.

11. Chen W, Wang $H$, Chen $H$, Liu S, Lu H, et al. (2014) Clinical significance and detection of microRNA-21 in serum of patients with diffuse large B-cell lymphoma in Chinese population. Eur J Haematol 92: 407-412.

12. Fiammengo $R$ (2017) Can nanotechnology improve cancer diagnosis through miRNA detection ? Biomark Med 11: 69-86.

13. Degliangeli F, Pompa PP, Fiammengo R (2014) Nanotechnology-based strategies for the detection and quantification of microRNA. Chem A Eur $\mathrm{J} 20$ : 9476-9492.

14. Lingam S, Beta M, Dendukuri D, Krishnakumar S (2014) A focus on microfluidics and nanotechnology approaches for the ultra sensitive detection of microRNA. Microrna 3: 18-28.

15. Válóczi A, Hornyik C, Varga N, Burgyán J, Kauppinen S, et al. (2004) Sensitive and specific detection of microRNAs by northern blot analysis using LNAmodified oligonucleotide probes. Nucleic Acids Res 32: e175.

16. Li W, Ruan K (2009) MicroRNA detection by microarray. Anal Bioanal Chem 394: 1117-1124.

17. Benes V, Castoldi M (2010) Expression profiling of microRNA using real-time quantitative PCR, how to use it and what is available. Methods 50: 244-249.

18. Costa V, Aprile M, Esposito R, Ciccodicola A (2012) RNA-Seq and human complex diseases: recent accomplishments and future perspectives. Eur $\mathrm{J}$ Hum Genet 21: 134-142.

19. Hunt EA, Broyles D, Head T, Deo SK (2015) MicroRNA Detection: Current Technology and Research Strategies. Annu Rev Anal Chem 8: 150514142349001

20. Arata H, Komatsu H, Hosokawa K, Maeda M (2012) Rapid and Sensitive MicroRNA Detection with Laminar Flow-Assisted Dendritic Amplification on Power-Free Microfluidic Chip. PLoS One 7.

21. Potrich C, Vaghi V, Lunelli L, Pasquardini L, Santini GC, et al. (2014) OncomiR detection in circulating body fluids: a PDMS microdevice perspective. Lab Chip 14: 4067-4075

22. Santini GC, Potrich C, Lunelli L, Vanzetti L, Marasso S, et al. (2016) Implementation of a PDMS microdevice for the improved purification of circulating microRNAs. Eur Cells Mater 32

23. Halldorsson S, Lucumi E, Gómez-Sjöberg R, Fleming RMT (2015) Advantages and challenges of microfluidic cell culture in polydimethylsiloxane devices. Biosens Bioelectron 63: 218-231.

24. Mills CA, Martinez E, Errachid A, Gomila G, Samsó A, et al. (2005) Small Scale Structures: The Fabrication of Polymeric Nanostructures for Biomedical Applications Using Pattern Replication Techniques. Contrib to Sci 3: 47-56.
25. Angeli E, Mussi V, Fanzio P, Manneschi C, Repetto L, et al. (2014) Micro and nanofluidic platforms for advanced diagnostics. Edorium J Nanotechnol 1-7.

26. Assender H, Bliznyuk V, Porfyrakis K (2002) How surface topography relates to materials' properties. Science 297: 973-976.

27. El-Khoury V, Pierson S, Kaoma T, Bernardin F, Berchem G (2016) Assessing cellular and circulating miRNA recovery: the impact of the RNA isolation method and the quantity of input material. Sci Rep 6: 19529.

28. Shihara RI, Chino YU, Osokawa KH, Aeda MM, Ikuchi AK (2017) Preparation of a Surface-functionalized Power-free PDMS Microchip for MicroRNA Detection Utilizing Electron Beam- induced Graft Polymerization. Anal Sci 33: 197-202.

29. Larrea E, Sole C, Manterola L, Goicoechea I, Armesto M, et al. (2016) New concepts in cancer biomarkers: Circulating miRNAs in liquid biopsies. Int $\mathrm{J}$ Mol Sci 17.

30. Chen X, Ba Y, Ma L, Cai X, Yin Y, et al. (2008) Characterization of microRNAs in serum: a novel class of biomarkers for diagnosis of cancer and other diseases. Cell Res 18: 997-1006.

31. Vaghi V, Potrich C, Pasquardini L, Lunelli L, Vanzetti L, et al. (2016) On-chip purification and detection of hepatitis $C$ virus RNA from human plasma. Biophys Chem 208: 54-61.

32. Jin $Y$, Zhang $Y$, Wu Y, Menon N V, Hian G, et al. (2015) Combinatorial effect of substratum properties on mesenchymal stem cell sheet engineering and subsequent multi-lineage differentiation. Acta Biomater 23: 52-62.

33. Li Z, Han W, Kozodaev D, Brokken-Zijp JCM, De With G, et al. (2006) Surface properties of poly(dimethylsiloxane)-based inorganic/organic hybrid materials. Polymer 47: 1150-1158.

34. Chen H, Meisburger SP, Pabit SA, Sutton JL, Webb WW, et al. (2012) Ionic strength-dependent persistence lengths of single-stranded RNA and DNA. Proc Natl Acad Sci 109: 799-804.

35. Hyeon C, Dima RI, Thirumalai D (2006) Size, shape, and flexibility of RNA structures. J Chem Phys 125.

36. Bin X, Sargent EH, Kelley SO (2010) Nanostructuring of Sensors Determines the Efficiency of Biomolecular Capture. Anal Chem 82: 5928-5931.

37. Pasquardini L, Potrich C, Quaglio M, Lamberti A, Guastella S, et al. (2011) Solid phase DNA extraction on PDMS and direct amplification. Lab Chip 11 4029.

38. Nečas D, Klapetek P (2012) Gwyddion: an open-source software for SPM data analysis. Open Phys 10: 181-188.

39. Fanzio P, Manneschi C, Angeli E, Mussi V, Firpo G, et al. (2012) Modulating DNA translocation by a controlled deformation of a PDMS nanochannel device. Sci Rep 2: 791.

40. Kuncová-Kallio J, Kallio PJ (2006) PDMS and its suitability for analytical microfluidic devices. Conf Proc IEEE Eng Med Biol Soc 1: 2486-9.

41. Azimzadeh $M$, Rahaie $M$, Nasirizadeh N, Daneshpour M, Naderi-Manesh $H$ (2017) Electrochemical miRNA Biosensors : The Benefits of Nanotechnology. Nanomedicine Res J 2: 36-48. 